\title{
CHAOS EXPANSIONS AND LOCAL TIMES
}

\author{
DaVid NUALART AND JOSEP VIVES
}

\begin{abstract}
In this note we prove that the Local Sime at zero for a multiparametric Wiener process bclongs to the Sobolev space $\mathbb{D}^{k-\frac{1}{2}-\iota_{1} 2}$ for any $\epsilon>0$. We do this computing its Wiencr clatos expansion. We sec also that this expansion converges almost surely. Finally, using the same lechnique we prove sitnilar resutts for a renomalized locil Time for the autointersect,ions of a planar Brownian motion.
\end{abstract}

\section{Introduction and notations}

In this note we first obtain the Wiener chaos decomposition of the local time at zero for a multiparameter Wiener process. We also show that the Wiener chaos series converges almost surely, and the local time belongs to the Sobolev space $\mathbf{D}^{k-1 / 2-\varsigma, 2}$, for any $\epsilon>0$, where $k$ is the number of parameters of the Wiener process. 'T'he last part of the paper is devoted to show the existence of a renormalized local time for the autointersections of a planar Brownian motion (Varadhan renormalization), by means of the Wiener chnos expansion.

Let $(T, B, \mu)$ be a $\sigma$-finite atomless measure space. We will denote by $H$ the Hilbert space $L^{2}(T, \mathcal{B}, \mu)$ which is assumed to the separable. Let $W=\{W(h), h \in H\}$ be a zero-mean Gaussian process with covariance function $E[W(f) W(g)]=\langle f, g\rangle_{H}$ defined on some probability space $(\Omega, \mathcal{F}, P)$. We will suppose that $\mathcal{F}$ is the $\sigma$-field generated by $\{W(h), h \in$ $H\}$. 1t is well-known that any square-integrable functional on $\Omega$ has an ortlogonal decomposition of the form

$$
F=E[F]+\sum_{n=1}^{\infty} I_{n}\left(f_{n}\right)
$$

where $f_{n} \in L_{s}^{2}\left(T^{n}\right)$ (symmetric square integrable kernel), and $I_{n}$ denotes the multiple Wiener-Itô stochastic integral. 
In this framework we can consider the derivative operator $D$ which acts on multiple stochastic integrals in the following form,

$$
D_{t} I_{n 1}\left(f_{n}\left(t_{1}, \ldots, t_{n 2}\right)\right)=n I_{n-1}\left(f_{n}\left(t_{1}, \ldots, t_{n-1}, t\right)\right)
$$

for $n \geq 1, t \in T$. We can introduce the Sobolev spaces $0^{\alpha, 2}$ for $\alpha \in \mathbb{R}$, as it is done in [11]. A functional $F \in L^{2}(\Omega)$ with the development (1) belongs to $\mathbb{Q}^{\alpha, 2}$ if and only if

$$
\sum_{n \geq 1} n !(1+n)^{\alpha}\left\|f_{n}\right\|_{2}^{2}<\infty
$$

Set $\mathbb{D}^{\infty, 2}=\cap_{\alpha \in \mathbb{R}} \mathbb{D}^{\alpha, 2}$ and $\mathbb{D}^{\alpha-2}=\cap_{\gamma<\alpha} \mathbb{D}^{\gamma, 2}$ for all $\alpha \in \mathbb{R}$.

\section{Preliminaries}

Let us first recall the Stroock formula (cf. [8]) that gives the Wiener chaos decomposition of a functional $F$ belonging to $0^{\infty, 2}$ :

$$
F=\sum_{n=0}^{\infty} \frac{1}{n !} I_{n}\left(E\left[D^{n} F\right]\right)
$$

We will also make use of the Hermite polynomials. For each $n \geq 0$, we will denote by $H_{n}(x)$, the $n$th Hermite polynomal defined by

$$
H_{n}(x)=\frac{(-1)^{n}}{\sqrt{n !}} e^{x^{2} / 2} \frac{d^{n}}{d x^{n}}\left(e^{-x^{2} / 2}\right), \quad n \geq 0 .
$$

Let $p_{\varepsilon}(x)$ be the contered Gaussian kernel with variance $\varepsilon>0$. The following equality, which follows immediatcly from (3), relates the derivatives $p_{\varepsilon}^{(n)}(x)$ with the Hermite polynomials:

$$
p_{\varepsilon}^{(n)}(x)=(-1)^{n} \sqrt{n !} \varepsilon^{-n / 2} p_{\varepsilon}(x) H_{n}\left(\frac{x}{\sqrt{\varepsilon}}\right), \quad n \geq 1 .
$$

\section{Lemma 1.1.}

Let $Y$ be a random variable with distribution $N\left(0, \sigma^{2}\right)$. Then

$$
E\left[H_{2 m}(Y)\right]=\frac{\sqrt{2 m !}\left(\sigma^{2}-1\right)^{m}}{2^{n} m !},
$$

and $E\left[H_{n}(Y)\right]=0$ if $n$ is odd.

Proof:

It follows easily from the explicit formula for Hermite polynomials:

$$
H_{n}(x)=\sqrt{n !} \sum_{k=0}^{[n / 2} \frac{(-1)^{k} x^{n-2 k}}{k !(n-2 k) ! 2^{k}},
$$

and the moments of a Gaussian random variable, $E\left[Y^{2 m}\right]=\frac{(2 m) !}{m ! 2^{m}}$. 


\section{Lemma 1.2.}

Let $\left\{F_{\varepsilon}\right\}_{\varepsilon}>0$ be a family of square integrable random warables with the expansions

$$
F_{\varepsilon}=\sum_{n=0}^{\infty} I_{n}\left(f_{n}^{\varepsilon}\right), \quad f_{n}^{\epsilon} \in L_{s}^{2}\left(T^{n}\right) .
$$

Assume that

i) $f_{n}^{\epsilon}$ converges in $L^{2}\left(T^{n}\right)$, when $\varepsilon \downarrow 0$, to some function $f_{n} \in L_{s}^{2}\left(T^{n}\right)$.

$$
\sum_{n=0}^{\infty} \sup _{\varepsilon}\left\{n !\left\|f_{n}^{\varepsilon}\right\|_{2}^{2}\right\}<\infty .
$$

Then the family $F_{\varepsilon}$ converges in $L^{2}(\Omega)$ to $F=\sum_{n=0}^{\infty} I_{n}\left(f_{n}\right)$.

\section{Proof:}

It is an immediate consequence of the Lebesgue dominated convergence theorem.

\section{Chaos expansion of $\delta_{0}(W(h))$}

Let $\delta_{0}$ be the Dirnc delta function at, zero. We car corsider $\delta_{0}(W(h))$ as a distribution on the Wiener space in the sense of Watarabe (cf. [11]). Using the integration by parts formula on the Wiener space one can show that $p_{\varepsilon}(W(h))$ converges in $\mathbb{D}^{-1,2}$ to $\delta_{0}(W(h)$ ) (see [5]). We will first. compute the Wiener chetos expansion of $p_{\varepsilon}(W(h))$, and from it we will deduce the expansion of $\delta_{0}(W(h))$. By formulas (2) and (3) we have

$$
\begin{aligned}
& p_{\varepsilon}(W(h))=\sum_{n=0}^{\infty} \frac{1}{n !} E\left[p_{\varepsilon}^{(n)}(W(h))\right] I_{n}\left(h^{\otimes n}\right) \\
& =\sum_{n=0}^{\infty} \frac{(-1)^{n t}}{\sqrt{n !} \varepsilon^{n / 2}} E\left[p_{\varepsilon}(W(h)) H_{n}\left(\frac{W(h)}{\sqrt{\varepsilon}}\right)\right] I_{n}\left(h^{\otimes n}\right) .
\end{aligned}
$$

The expectation appearing in the above formula varnishes if $n$ is odd because $p_{\varepsilon}$ and $H_{n}$ are even functions. On the other haud, using Lemma 1.1 for $n=2 m$ we obtain

$$
\begin{aligned}
\int_{\mathbb{R}} H_{2 m} & \left(\frac{x}{\sqrt{\varepsilon}}\right) p_{\varepsilon}(x) p_{\left\|h_{h}\right\|^{2}}(x) d x \\
& =\left(2 \pi\left(\|h\|^{2}+\varepsilon\right)\right)^{1 / 2} \int_{\mathbb{R}} I_{2 m}\left(\frac{x}{\sqrt{\varepsilon}}\right) p_{\varepsilon\left\|k_{\|}\right\|^{2} /\left(\varepsilon-1-\|h\|^{2}\right)}(x) d x \\
& =\left(2 \pi\left(\|h\|^{2}+\varepsilon\right)\right)^{-1 / 2} \frac{\sqrt{2 m !}}{2^{m} m !}\left(\frac{-\varepsilon}{\|h\|^{2}+\varepsilon}\right)^{m} .
\end{aligned}
$$


Finally, from (6) and (7), we get the following cxpansion

$$
p_{\varepsilon}(W(h))=\sum_{m=0}^{\infty} \frac{(-1)^{m} I_{2 \pi n}\left(h^{\otimes 2 m}\right)}{\sqrt{2 \pi} 2^{m} m !\left(\|h\|^{2}+\varepsilon\right)^{m+1 / 2}} .
$$

Letting $\varepsilon$ tend to zero we deduce the Wiener chaos expansion of $\delta_{0}(W(h))$ :

$$
\delta_{0}(W(h))=\sum_{m=0}^{\infty} \frac{(-1)^{m} I_{2 m}\left(h^{\otimes 2 n}\right)}{\sqrt{2 \pi} 2^{m n} r n !\|h\|^{2 n+1}} .
$$

This series does not converge in $L^{2}(\Omega)$, because

$$
\left\|\delta_{0}(W(h))\right\|_{2}^{2}=\sum_{r n=0}^{\infty} \frac{(2 m) !}{2^{2 m}(m !)^{2} 2 \pi\|h\|^{2}}=\infty \text {, }
$$

by the Striling formula. Observe that from (9) and (10) we obtain

i) $\delta_{0}(W(h)) \in \mathbb{D}^{-1 / 2-, 2}$

ii) $\delta_{0}(W(h)) \notin \mathbb{D}^{-1 / 2,2}$;

and the series (9) converges in the norm of the space $\mathbb{D}^{-1 / 2-\epsilon, 2}$, for any $\epsilon>0$.

Remark. More generally we can obtain the chaos expansion of $\delta_{x}(W(h))$ when $x \neq 0$ :

$$
\delta_{x}(W(h))=\sum_{n=0}^{\infty} p_{\left\|h^{2}\right\|^{2}}(x) H_{n}\left(\frac{x}{\|h\|}\right) \frac{I_{n}\left(h^{\otimes n}\right)}{\| h^{n} \sqrt{n !}} .
$$

\section{Wiener chaos expansion for the local time of a multiparametric Wiener process}

In this section we will assume that $T$ is $[0,1]^{k}$, with $k \geq 1$. Then $W=\{W(\underline{t}), \underline{t} \in T\}$ will be the standard Wiener process on $T$. We will denote by $[0, t]$ the rectangle $\left[0, t_{1}\right] \times \cdots \times\left[0, t_{k}\right]$, where $\underline{t}=\left(t_{1}, \ldots, t_{k}\right)$. We will also set $|\underline{\underline{t}}|=t_{1} \cdot \ldots \cdot t_{k}$.

The local time of $W$ can be formally defined as

$$
L(\underline{\underline{t}}, x)=\int_{[0, \underline{\underline{t}}]} \delta_{x}\left(W_{\underline{s}}\right) d \underline{s}, \quad \underline{t} \in T, \quad x \in \mathbb{R} .
$$

Although for any fixed $\underline{s}, \delta_{x}\left(W_{\underline{s}}\right)$ is not an ordinary random variable but a distribution on the Wiener space, it turns out that the integral 
in (11) has a smoothing eflect, and $L(\underline{t}, x)$ is a well-defined random variable for any fixed point $\underline{l}$, nol on the axes. We will restrict our analysis to the case $x=0$, and we will set $L(\underline{t})=L(\underline{t}, 0)$. We know that $L(\underline{t})=\int_{0, \underline{t} !} \delta_{0}\left(W_{y}\right) d \underline{s}$ can be obtained as the $L^{2}$-limit of

$$
L_{\varepsilon}(\underline{t})=\int_{[0, \underline{\underline{\varepsilon}}]} p_{\varepsilon}\left(W_{\underline{s}}\right) d \underline{s}
$$

when $\varepsilon$ tends to 0 (see, for instance, [2]). In the next theorem we will compute the Wiener chaos exparision of $L(t)$.

\section{Theorem 3.1.}

We have that $L(\underline{L})$ belongs to the space $\mathbb{D}^{k-\frac{1}{2}-, 2}$, for any point $t$ not on the axes, and it holds that

$$
\begin{aligned}
& L(\underline{l})=\sum_{m=0}^{\infty} \frac{(-1)^{\pi+} 2^{k}}{\sqrt{2 \pi} 2^{m} m !(1-m)^{k}} I_{2 \pi} \\
& {\left[\prod_{i=1}^{k}\left(\left(t_{i}\right)^{(1-m) / 2}-\left(t_{1, i} \vee \cdots \vee t_{2 m, i}\right)^{(1 \cdots m) / 2}\right)\right] .}
\end{aligned}
$$

Moreover, $L(t)$ does not belong to $\mathbb{D}^{k-\frac{1}{2} 1^{2}}$.

\section{Proof:}

We will first compute the Wiener chaos expansion of $L_{\epsilon}(\underline{t})$ applying the results of the previous section. From (8) and (11) we obtain

$$
L_{\varepsilon}(\underline{t})=\sum_{m=0}^{\infty} \frac{(-1)^{m}}{\sqrt{2 \pi} 2^{m !} m !} \int_{[0, \underline{\ell}]} \frac{I_{2 m}\left(\mathbf{1}_{[0, \underline{s}]}^{\otimes 2 m}\right)}{(\mid \underline{s} ;+\varepsilon)^{m+1 / 2}} d \underline{s} .
$$


Then the series $\sum_{n=1}^{\infty} X_{n}$ converges a.s.

As a consequence of this theorem, if $F$ is a square integrable random variable with the development. (1), and

$$
\sum_{n=0}^{\infty} n !(\log n)^{2}\left\|f_{n}\right\|_{2}^{2}<\infty
$$

then the Wiener chaos expansion (1) converges a.s. In particular the condition (14) is satisfied if $F$ belongs to the Sobolev space $\mathbb{B}^{5,2}$ for any $\epsilon>0$. Consequently, applying Theorem 3.1 , and the above criterion (14), we deduce the amost sure convergence of the Wiener chaos expansion of the local time of the multiparameter Wiener process.

\section{Renormalized local time for the autointersections of a planar Brownian motion}

Consider now $W=\left\{\left(W_{t}^{1}, W_{t}^{2}\right), t \in[0,1]\right\}$ a standard planar Brownian motion. Let us write $\left[X^{\prime}\right]=X-E(X)$ for any integrable random variable $X$. It is known from $[6]$ that

$$
L_{\varepsilon}=\int_{0<s<t<1}\left[p_{\varepsilon}\left(W_{t}^{1}-W_{s}^{\prime}\right) p_{\varepsilon}\left(W_{t}^{2}-W_{s}^{2}\right)\right] d s d t
$$

converges in $L^{2}(\Omega)$, as $\varepsilon$ tends to zero. The purpose of this section is to give a new proof of this fact by means of the results obtained on Section 2.

\section{Theorem 4.1.}

The family of random variables $L_{\varepsilon}$ converges as $\varepsilon$ tends to zero, in $\mathbb{D}^{1 / 2-\delta, 2}$, for any $\delta>0$. In particular; this implies the convergence in $L^{2}(\Omega)$.

Proof:

Set $\Delta=(s, t]$. Applying the results of Section 2, we have (16)

$$
L_{\varepsilon}=\sum_{n=1}^{\infty} \frac{(-1)^{n}}{2 \pi 2^{n}} \sum_{\ell \rightarrow p=n} \frac{1}{\ell ! p !} \int_{0<s<t<1} \frac{I_{2 \ell}^{1}\left(1_{\Delta}^{\otimes 2 \ell}\right) I_{2 p}^{2}\left(1_{\Delta}^{\otimes 2 p}\right)}{(|\Delta|+\varepsilon)^{n+1}} d s d t
$$

where $I_{2 \ell}^{1}$ and $I_{2 p}^{2}$ denote, respectively, the multiple stochastic integrals with respect to the Brownian motions $W^{1}$ and $W^{2}$. When $n$ varies the 
terms appearing in the above sum are orthogonal. The square of the $L^{2}$-norm of the nith term is given by

$$
\begin{aligned}
& \frac{1}{(2 \pi)^{2} 2^{2 n}} \sum_{\ell+p=n} \frac{1}{(\ell !)^{2}(p !)^{2}} \\
& \int_{\substack{\ell<t \\
\ell<\nu}} \frac{E\left[I_{2 \ell}^{1}\left(1_{\Delta}\right) I_{2 \ell}^{1}\left(I_{\Delta^{*}}\right)\right] E\left[I_{2 p}^{2}\left(1_{\Delta}\right) I_{2 p}^{2}\left(1_{\Delta^{*}}\right)\right]}{\left[(|\Delta|+\varepsilon)\left(\left|\Delta^{*}\right|+\varepsilon\right)\right]^{n+1}} d s d t d u d v
\end{aligned}
$$

where $\Delta^{*}=(u, v]$. We can estimate this term by

$$
\begin{aligned}
& \frac{(2 n) !}{(2 \pi)^{2} 2^{2 n}(n !)^{2}} \sum_{\ell+p=n}\left(\frac{n !}{\ell ! p !}\right)^{2} \int_{\substack{s<! \\
u<v}} \frac{(2 \ell) !(2 p) !\left(1_{\Delta}, 1_{\Delta}\right)^{2 n}}{(2 n) !\left(|\Delta|\left|\Delta^{*}\right|\right)^{n+1}} d s d t d u d v \\
& =\frac{(2 n) !}{(2 \pi)^{2} 2^{2 n}(n !)^{2}}\left[\sum_{\ell+p=n} \frac{\left(\begin{array}{c}
n \\
\ell
\end{array}\right)\left(\begin{array}{c}
n \\
p
\end{array}\right)}{\left(\begin{array}{c}
2 n \\
2 \ell
\end{array}\right)}\right] \int_{\substack{y<t \\
u<v}} \frac{\left|\Delta \cap \Delta^{*}\right|^{2 n}}{\left(|\Delta|\left|\Delta^{*}\right|\right)^{n+1}} d s d t d u d v .
\end{aligned}
$$

Observe that

$$
\sum_{\ell+p=n} \frac{\left(\begin{array}{c}
n \\
\ell
\end{array}\right)^{2}}{\left(\begin{array}{c}
2 n \\
2 \ell
\end{array}\right)} \leq(n+1) \max _{0 \leq \ell \leq n} \frac{\left(\begin{array}{l}
n \\
\ell
\end{array}\right)^{2}}{\left(\begin{array}{c}
2 n \\
2 \ell
\end{array}\right)} \leq n+1 .
$$

On the other hand we claim that

$$
\int_{\substack{s<t \\ u<v}} \frac{|(s, t] \cap(u, v]|^{2 n}}{(t-s)^{n+1}(v-u)^{n+1}} d s d t d u d v \leq \frac{3}{n^{2}} .
$$

In order to show (18) we will decompose the integral by considering the different positions of $s, t, u$ and $v$. We have that the left hand side of (18) is equal to

$$
\begin{aligned}
2 \int_{u<s<v<t} \frac{(v-s)^{2 n}}{(t-s)^{n+1}(v-u)^{n+1}} d s d t d u d v \\
+2 \int_{u<s<t<v} \frac{(t-s)^{n-1}}{(v-u)^{n+1}} d s d t d u d v .
\end{aligned}
$$

The second summand in (19) can be estimated as follows

$$
\frac{2}{n} \int_{u<s<v} \frac{(v-s)^{n}}{(v-u)^{n+1}} d u d s d v=\frac{1}{n(n+1)} \leq \frac{1}{n^{2}} .
$$


For the first term, we have

$$
\begin{aligned}
& \frac{2}{n} \int_{s<v<t} \frac{(v-s)^{n}}{(t-s)^{n+1}} d s d t d v-\frac{2}{n} \int_{v<v<t} \frac{(v-s)^{2 n}}{(t-s)^{n+1} v^{n}} d s d v d t \\
& =\frac{1}{n(n+1)}-\frac{2}{n^{2}} \int_{s<v} \frac{(v-s)^{2 n}}{(1-s)^{n} v^{n}} d s d v+\frac{2}{n^{2}} \int_{s<v} \frac{(v-s)^{n}}{v^{n}} d s d v \\
& =\frac{1}{n(n+1)}+\frac{2}{n^{2}} \int_{s<v} \frac{(v-s)^{n}}{v^{n}}\left[1-\frac{(v-s)^{n t}}{(1-s)^{n t}}\right] d s d v \\
& \leq \frac{1}{n(n+1)}+\frac{1}{n^{2}} \leq \frac{2}{n^{2}},
\end{aligned}
$$

which completes the proof of (18). Therefore the square of the $L^{2}$ norm of each lerm of (16) can be estimated by

$$
\frac{(2 n) !}{(2 \pi)^{2} 2^{2 n}(n !)^{2}} \frac{3(n+1)}{n^{2}}
$$

which is equivalent to a constart times $n^{-3 / 2}$. Then Lemma 1.2 allows to complete the proof of the theorem.

\section{References}

1. N. BOULEAU AND F. HIRSCH, "Ditichlet forms and analysis on Wiener space," Walter de Gruyter, 1991.

2. D. Geman AND J. Horowitz, Occupation densitics, Annals of Probability 8 (1980), 1-67.

3. J. F. LE GALL, "Sur le temps local d'intersection du mouvement brownien plan ct la méthode de renormalisation de Varadhan," Sem. Prob. XIX, Lecture Notes in Math. 1123, 1984, pp. 314-331.

4. D. NUALART AND E. PARDOUX, Stochastic calculus with anticipating integrands, Prob. Theory and Rel. Fields 78 (1988), 535-581.

5. D. Nunlar'T AND J. VIVes, Smoothness of Brownian local times and related functionals, Preprint.

6. J. Ros:N, "A renormalized local time for multiple intersections of planar Brownian motion," Sern Prob. XX, Lecture Notes in Math. 1204, 1985, pp. 515-531.

7. W. STou'r, "Almost sure converyence," Academic Press, 1984.

8. D. W. STroock, "Homogencons Chaos revisited," Sem. Prob. XXI, Lecture Notes in Math. 1247, 1987, pp. 1-7. 
9. H. SuGiTA, Sobolev spaces of Wiener functionals and Malliavin's calculus, J. Math. Kyoto Univ. 25, 1 (1985), 31-48.

10. J. B, Walshi, The local time of the brownian sheet, Astérisque 52, 53 (1978), 47-61.

11. S. WATANABE, "Lectures on stochastic differential equations and Malliavin Calculus," Springer, 1984.

David Nualart:

Facultat de Matemàtiques

Universitat de Barcelona

Gran Via, 585

08007 Barcelona

SPAIN
Josep Vives:

Departament de Matemàtiques

Universitat Autònoma de Barcelona

08193 Bellaterra (Barcelona)

SPAIN

Rebut el 2 de Marৎ de 1992 